\title{
Regular and irregular wave impacts on floating body
}

\author{
Yong Li, Mian Lin* \\ Institute of Mechanics, Chinese Academy of Sciences, No. 15 West Road, North 4th Ring Road, Beijing 100190, China
}

\section{A R T I C L E I N F O}

\section{Article history:}

Received 13 January 2011

Accepted 2 January 2012

Editor-in-Chief: A.I. Incecik

Available online 25 January 2012

\section{Keywords:}

Floating body

Irregular wave

Wave impact

Numerical wave tank

Finite volume method

\begin{abstract}
A B S T R A C T
Fully nonlinear wave-body interactions for a stationary floating structure under regular and irregular waves for different water depths, wave heights and periods are studied in a 2-D numerical wave tank. The tank model is based on Reynolds-averaged Navier-Stokes equations and renormalization group $k-\varepsilon$ model. The equations are discretized based on the finite volume method. The pressure implicit splitting of operators scheme is employed to treat the pressure-velocity coupling and a compressive interface capturing scheme is used to capture the free surface on mashes of arbitrary topology. The calculated results for regular wave simulation, irregular wave propagation and wave impacts on floating body are compared with the theoretical/experimental data and the numerical results agree well with analytical/ experimental solutions. The mean and maximum wave impacts, including rotational moment, on body are obtained. The effects of water depth, wave height and period on forces and moment have been investigated and the calculated results for irregular waves are compared with those induced by regular waves.
\end{abstract}

(c) 2012 Elsevier Ltd. All rights reserved.

\section{Introduction}

Recently, many types of floating structures, such as floating breakwater, jacket platform and man-made island, have been used in coastal and ocean engineering. The safety of these structures has a significant relation to the wave impacts. The structures usually suffer from strong nonlinear loadings under waves, especially under irregular waves. Therefore, prediction of nonlinear regular and irregular wave forces on floating structures is an important topic.

There are many correlative studies on the interactions between the water waves and structures with different types, including the fully submerged structures (Clement and Mas, 1995; Boo, 2002; Koo et al., 2004; Vengatesan et al., 2006, etc.), vertical cylinders/ plates ( $\mathrm{Li}$ and Lin, 2001; Pradip and Sukamal, 2006; Wang and Wu, 2010, etc.), the surface-piercing bodies (Nojiri and Murayama, 1975; Tanizawa and Minami, 1998; Fang and Chen, 2001; Koo and Kim, 2007a, 2007b; Li and Lin, 2010, etc.) and so on.

Many researchers studied the interaction problem based on theory and experimental analyses. Pradip and Sukamal (2006) introduced a solution of shallow water wave force, using small amplitude linear wave theory on two-dimensional (2-D) vertically submerged circular thin plates under different configurations. The total horizontal force and moment with respect to the wave amplitude were obtained at different water depths and

\footnotetext{
* Corresponding author. Tel.: +86108254 4206.

E-mail addresses: linmian@imech.ac.cn, liyong@imech.ac.cn (M. Lin).
}

wave periods. Hanssen and Torum (1999) experimentally studied the breaking wave forces on tripod concrete structure on shoal using Morison's equation. Ren and Wang (2003) studied the irregular wave slamming on structure members with large dimension in the splash zone. The time-domain and frequencydomain analyses results of the irregular wave impact pressure on the subface of the structure were presented.

Some of the other researchers investigated the interaction problem using numerical wave tank. In Boo's work (2002), a time-domain numerical scheme was used to simulate the linear irregular waves in numerical tank and the linear and nonlinear irregular wave diffraction forces acting on a submerged structure was predicted. Koo and Kim (2007a) studied the wave body interactions for stationary floating single and double bodies using a potential-theory-based fully nonlinear 2-D numerical wave tank. Li and Lin (2010) investigated the fully nonlinear wave-body interactions for a surface-piercing body in finite water depth with flat/slop bottom topography. A 2-D numerical regular wave tank was built, which mainly based on the spatially averaged NavierStokes equations and the $k-\varepsilon$ model was used to simulate the turbulence of flow. Clauss et al. (2010) studied the fully nonlinear interactions between water waves and vertical cylinder arrays in a numerical tank, which based on a finite element method (FEM).

Among studies presented above, the problems on interaction between water waves and floating bodies have been interested recently. The regular wave and/or current had been considered by some authors (Koo and Kim, 2007a, 2007b; Li and Lin, 2010, etc.). It is important to understand nonlinear interactions between irregular waves and surface-piercing structures, compared with 
regular waves. In present paper, using a fully nonlinear 2-D numerical wave tank, the wave forces and rotational moment on a floating structure are investigated for regular and irregular waves at different water depths, wave heights and periods.

With the development of computer technology and computation algorithm, the numerical wave tank has been developed to be a promising tool to investigate various wave-related problems. In order to study the problem of wave propagation, a fully nonlinear numerical wave tank was presented by Zhang et al. (2006). In their studies, desingularized boundary integral equation method was coupled with the mixed Eulerian-Lagrangian formulation. Li (2008) described a numerical tank for regular and irregular wave propagation based on the Navier-Stokes equations and a spatial fixed $\sigma$-coordinate was used to transform the equations from the sea bed to the still water level.

In present works, the numerical tank is built based on Reynolds-averaged Navier-Stokes (RANS) equations and renormalization group (RNG) $k-\varepsilon$ two-equation model. The wave tank is verified firstly and the simulated results are compared with the theoretical/experimental solutions. Then, mean and maximum wave forces and rotational moment are calculated. The force spectra are obtained by fast Fourier transform (FFT), though which the forces and moment are transformed from the timedomain to frequency-domain.

\section{Mathematical formulation}

\subsection{Governing equations}

The governing equations are the RANS equations, which can be written as follows:

$\frac{\partial}{\partial x_{j}}\left(u_{j}\right)=0$,

$\frac{\partial}{\partial t}\left(u_{i}\right)+\frac{\partial}{\partial x_{j}}\left(u_{j} u_{i}\right)=-\frac{1}{\rho} \frac{\partial p}{\partial x_{i}}+\frac{1}{\rho} \frac{\partial}{\partial x_{j}}\left[\mu_{e f f}\left(\frac{\partial u_{j}}{\partial x_{i}}+\frac{\partial u_{i}}{\partial x_{j}}\right)\right]-D_{i} u_{i}+g_{i}$

where $x_{j}(j=1,2)$ represents the coordinate component, $u_{j}$ is the fluid velocity, $p$ is the pressure, $\rho$ is the density, $g$ is the acceleration of gravity, $D_{i}$ is the damping coefficient and the damping term $D_{i} u_{i}$ is added to the momentum equation directly. $\mu_{\text {eff }}=\mu+\mu_{f}, \mu$ is the molecular viscosity, $\mu_{f}$ is the turbulent eddy viscosity, $\mu_{f}=C_{\mu} \rho k^{2} / \varepsilon . k$ is the turbulent kinetic energy, $\varepsilon$ is the turbulent energy dissipation rate. In present paper, the RNG $k-\varepsilon$ two-equation model is adopted to estimate the turbulence

$\frac{\partial}{\partial t}(\rho k)+\frac{\partial}{\partial x_{j}}\left(\rho u_{j} k\right)=\frac{\partial}{\partial x_{j}}\left[\alpha_{k} \mu_{e f f} \frac{\partial k}{\partial x_{j}}\right]+P_{k}-\rho \varepsilon$,

$\frac{\partial}{\partial t}(\rho \varepsilon)+\frac{\partial}{\partial x_{j}}\left(\rho u_{j} \varepsilon\right)=\frac{\partial}{\partial x_{j}}\left[\alpha_{\varepsilon} \mu_{e f f} \frac{\partial \varepsilon}{\partial x_{j}}\right]+C_{1 \varepsilon}^{*} P_{k} \frac{\varepsilon}{k}-\rho_{f} C_{2 \varepsilon} \frac{\varepsilon^{2}}{k}$

where

$P_{k}=\mu_{f}\left(\frac{\partial u_{i}}{\partial x_{j}}+\frac{\partial u_{j}}{\partial x_{i}}\right) \frac{\partial u_{i}}{\partial x_{j}}, C_{1 \varepsilon}^{*}=C_{1 \varepsilon}-\frac{\eta\left(1-\eta / \eta_{0}\right)}{1+\beta \eta^{3}}$,

$\eta=\left(2 S_{i j} S_{i j}\right)^{0.5} \frac{k}{\varepsilon}, S_{i j}=\frac{1}{2}\left(\frac{\partial u_{i}}{\partial x_{j}}+\frac{\partial u_{j}}{\partial x_{i}}\right)$.

The values of constants in RNG $k-\varepsilon$ model are shown in Table 1.

Table 1

Values of constants in turbulent model.

\begin{tabular}{llllllll}
\hline Constant & $C_{\mu}$ & $\alpha_{k}$ & $\alpha_{\varepsilon}$ & $C_{1 \varepsilon}$ & $C_{2 \varepsilon}$ & $\eta_{0}$ & $\beta$ \\
\hline Value & 0.0845 & 1.39 & 1.39 & 1.42 & 1.68 & 4.38 & 0.012 \\
\hline
\end{tabular}

In order to capture the water-air free surface, an Eulerian method named the volume of fluid (VOF) method is adopted. The equation for the volume fraction is

$\frac{\partial \alpha}{\alpha t}+\frac{\partial}{\partial x_{j}}\left(u_{j} \alpha\right)=0$

where $\alpha$ is the volume fraction of water and $1-\alpha$ represents the volume fraction of air. Volume fraction of each liquid is used as the weighting factor to get the mixture properties, for the density and molecule viscosity

$\rho=\alpha \rho_{w}+(1-\alpha) \rho_{a}$,

$\mu=\alpha \mu_{w}+(1-\alpha) \mu_{a}$

where $\rho_{w}$ and $\rho_{a}$ represent the density of water and air, respectively. $\mu_{w}$ and $\mu_{a}$ are molecule viscosity coefficient of water and air, respectively.

\subsection{Boundary conditions}

The entire study domain is shown in Fig. 1 . There are totally five types of boundary associated with the governing equations, including inlet, outlet, structure, bed and atmosphere. In addition, in order to absorb the wave energy reflection from end-wall and re-reflection from input boundary, artificial damping zones are located at the two ends of domain.

Boundary conditions associated with regular and irregular waves are prescribed along the inlet of computational domain. The pressure and turbulence quantities, such as $k$ and $\varepsilon$, are set to be zero normal gradients. To simulate wave-structure interaction steadily for a long time, a special damping scheme is employed in front of the inlet boundary, which can be used to prevent the rereflection from the left boundary. For the left damping zone, the velocity in this area is modified by $u_{i}=u_{i}^{m}+D_{i}^{\prime}\left(u_{i}-u_{i}^{m}\right)$ at the end of each time step. This damping scheme is employed to damp out only the reflected waves from the structure while preserving the incident waves. Similar treatment methods are used by many researches for wave-structure interaction problem (Koo and Kim, 2007a, 2007b; Li et al., 2007, etc.). In the damping scheme, $u_{i}^{m}$ is the theoretical wave velocity and $D_{i}^{\prime}$ is the dissipative coefficient, which can be written as (Troch and Rouck, 1998)

$D_{i}^{\prime}=\sqrt{1-\left(\frac{l-x}{l}\right)^{2}}$

where $l$ is the length of damping zone. Troch and Rouck (1998) found that the elliptic type damping function performs better than the linear type and the cosine type.

At the outlet, non-reflective boundary condition combines damping zone and the radiation boundary condition. The velocity is specified by Sommerfeld radiation condition (SRC), expressed by

$\frac{\partial u}{\partial x}+\frac{1}{c} \frac{\partial u}{\partial t}=0$

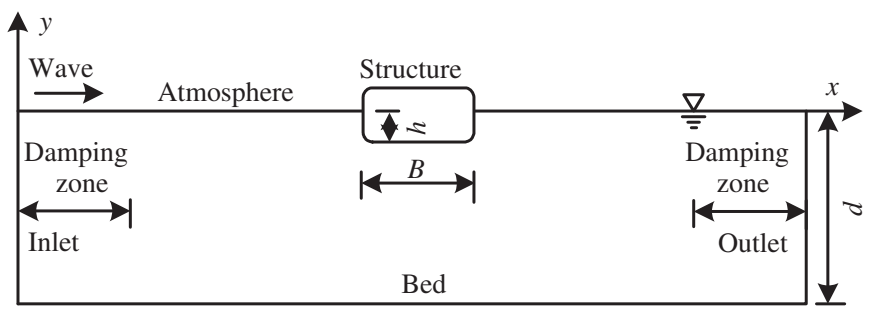

Fig. 1. Sketch of a fixed floating body. 
where $c$ is the wave propagation velocity at the end point of damping zone. As for the damping zone, $D_{i} u_{i}$ damping term is added to the momentum equation, showed as Eq. (2) and $D_{i}$ is expressed as

$D_{i}=\theta_{i} \sqrt{\frac{g}{d}}(n+1)\left(\frac{x-x_{0}}{l}\right)^{n}$

where $x_{0}$ is the distance from origin to the starting point of damping zone. $n$ and $\theta_{i}$ are the damping coefficients, $n=2, \theta_{i}=0.6$.

As for the structure wall and bed boundaries, no slip wall boundary condition and wall function are used. At the atmosphere boundary, the total pressure is set to zero and $k$ and $\varepsilon$ are set to zero normal gradient.

\subsection{Regular and irregular wave simulations}

The velocity vector of regular wave is specified by Stokes wave. The theoretical velocity of second-order Stokes regular wave can be expressed by

$u_{x}^{m}=\frac{a g H}{2 \omega} \frac{\operatorname{ch} a(z+d)}{c h a d} \cos (a x-\omega t)+\frac{3 \omega a H^{2}}{16} \frac{\operatorname{ch} 2 a(z+d)}{s^{4} a d} \cos 2(a x-\omega t)$,

$u_{y}^{m}=\frac{a g H}{2 \omega} \frac{\operatorname{sh} a(z+d)}{\operatorname{chad}} \sin (a x-\omega t)+\frac{3 \omega a H^{2}}{16} \frac{\operatorname{sh} 2 a(z+d)}{\operatorname{sh}^{4} a d} \cos 2(a x-\omega t)$,

where $H, \omega, a$ and $d$ are wave height, frequency, wave number and water depth, respectively. $x$ is the distance from origin to the wave-making point.

In the irregular wave simulation, the modified JONSWAP spectrum is chosen as the target spectrum, which can be expressed as follows (Goda, 1999):

$$
\begin{aligned}
& S(f)=\beta_{j} H_{1 / 3}^{2} T_{p}^{-4} f^{-5} \exp \left[-1.25\left(T_{p} f\right)^{-4}\right] \gamma^{\exp \left[-\left(f / f_{p}-1.0\right)^{2} /\left(2 \sigma^{2}\right)\right]}, \\
& \beta_{j}=\frac{0.06238 \times(1.094-0.01915 \ln \gamma)}{0.230+0.0336 \gamma-0.185 \times(1.9+\gamma)^{-1}},
\end{aligned}
$$

$T_{p}=\frac{T_{1 / 3}}{1.0-0.132(\gamma+0.2)^{-0.559}}$,

$\sigma= \begin{cases}0.07 & f \leq f_{p} \\ 0.09 & f>f_{p}\end{cases}$

where $H_{1 / 3}$ and $T_{1 / 3}$ are the significant wave height and period, respectively. $f$ is the frequency. $\gamma$ is the peak enhancement factor, $\gamma=3.3$. $T_{p}$ and $f_{p}$ denote the wave period and the frequency at the spectral peak, respectively.

The surface fluctuations and velocities of irregular wave in wave-making region can be obtained with

$\eta(t)=\sum_{i=1}^{N} \frac{H_{i}}{2} \cos \left(k_{i} x-\omega_{i} t+e_{i}\right)$,

$u_{x}^{m}=\sum_{i=1}^{N} \frac{a_{i} g H_{i}}{2 \omega_{i}} \frac{\cosh a_{i}(z+d)}{\cosh a_{i} h} \cos \left(a_{i} x-\omega_{i} t+e_{i}\right)$,

$u_{y}^{m}=\sum_{i=1}^{N} \frac{a_{i} g H_{i}}{2 \omega_{i}} \frac{\sinh a_{i}(z+d)}{\cosh a_{i} h} \sin \left(a_{i} x-\omega_{i} t+e_{i}\right)$

where $A_{i}=\sqrt{2 S\left(\widehat{\omega}_{i}\right) \Delta \omega_{i}}, \Delta \omega_{i}=\omega_{i}-\omega_{i-1}, \widehat{\omega}_{i}$ is a random variable number between $\omega_{i-1}-\omega_{i}, e_{i}$ and $N$ denote a random variable number between 0 and $2 \pi$ and the total number of sampling, respectively.

\subsection{Numerical implementation}

The equations are discretized based on the finite volume method (FVM). The central difference scheme and quadratic upwind interpolation of convective kinematics (QUICK) scheme are employed to represent the diffusion term and convection term, respectively. The preconditioning conjugate gradient (PCG) method is employed for solving the algebraic equations. The Navier-Stokes equations are solved numerically by the pressure correction method in two major steps. Firstly, the intermediate velocity field is obtained by solving the momentum equations. Secondly, the pressure correction is achieved by the Poisson equation, which can be derived by the combination of the continuity and the momentum equations. And then, the intermediate velocity is corrected by the divergence-free velocity, which can be obtained by the pressure correction. The pressure implicit splitting of operators (PISO) scheme is employed to treat the pressure-velocity coupling. $k$ and $\varepsilon$ equations are coupled in the scheme and solved by a segregated approach. For free surface simulation, a compressive interface capturing scheme for arbitrary meshes (CICSAM) is used to capture the fluid interfaces on mashes of arbitrary topology (Ubbink and Issa, 1999). Furthermore, the Courant number is used to obtain the time step at the every beginning of calculation cycle, defined as

$C_{n}=\frac{u_{i} \Delta t}{\Delta x_{i}}$

where $\Delta t$ is the time step and $\Delta x_{i}$ represents the mesh size.

The code is programmed based on the open source $C++$ CFD toolbox named open field operation and manipulation (OpenFOAM). OpenFOAM provides a fundamental platform to write new solvers for different problems and the tensorial approach and object oriented techniques are used. For example, a new direct simulation Monte Carlo (DSMC) foam has been written within the framework of OpenFOAM by Scanlon et al. (2010). In their cases, the new foam shows very good agreement with data provided by both analytical solutions and other contemporary DSMC codes.

In present work, a new two-fluid code, called waveFoam, has been written within the framework of OpenFOAM. The version used here is 1.7.1, which can be downloaded freely through the internet (OpenCFD 2010). In the waveFoam solver, the inflow boundary and two damping zones are added and the interface capturing scheme in OpenFOAM for two-phase flow is improved (Li and Lin, 2010).

\section{Numerical results and discussion}

\subsection{Model validation}

\subsubsection{Regular wave simulation}

The total length of the numerical tank is $8.0 \mathrm{~L}$ with sponge layers at its two ends, each with a length of $2.0 \mathrm{~L}$. The numerical case is performed with following input: $H=0.5 \mathrm{~m}, T=3.0 \mathrm{~s}$, $d=5.0 \mathrm{~m}$ and $C_{n}=0.1$. Fig. 2 shows the time series of wave profile at $4.0 \mathrm{~L}$ from the beginning of tank and the numerical results compared with the analytical solutions. It can be seen that the numerical results agree well with analytical solutions, which indicates that the two sponger layers can absorb the nonlinear wave effectively.

\subsubsection{Irregular wave simulation}

The irregular waves are simulated without structure for the whole repetition period of $120 T$. Fig. 3 illustrates the time series of irregular waves at $4.0 \mathrm{~L}$ from the beginning of tank. FFT is used to analyze the frequency spectrum. For the cases of $H_{1 / 3}=0.5 \mathrm{~m}$ and 


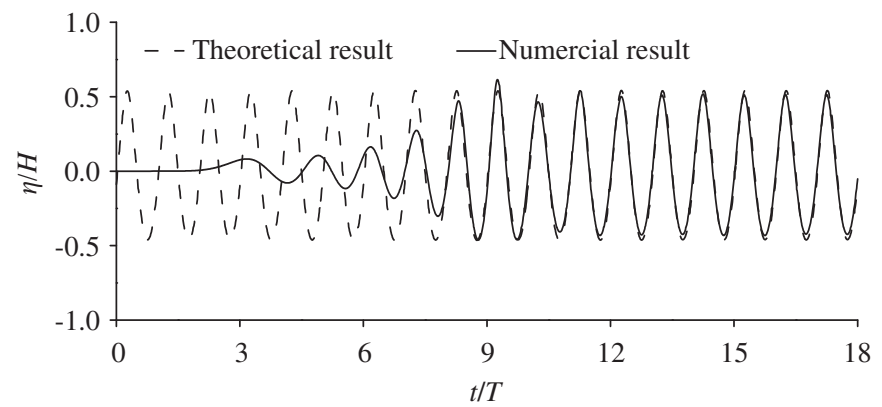

Fig. 2. Time series of regular wave profile.

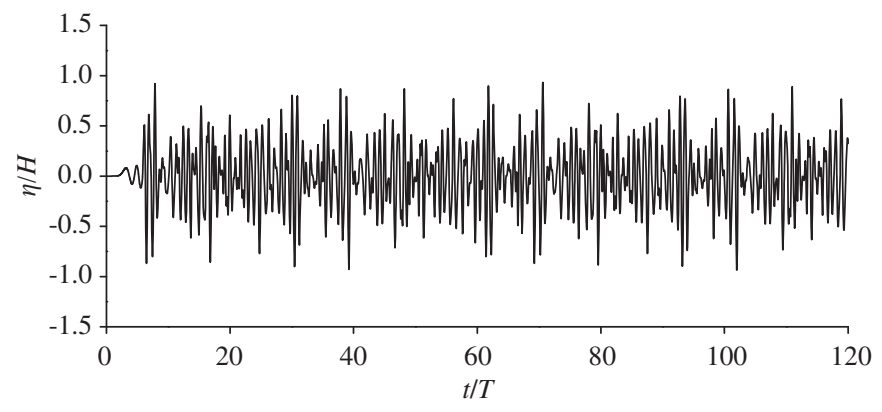

Fig. 3. Time series of irregular wave profile.

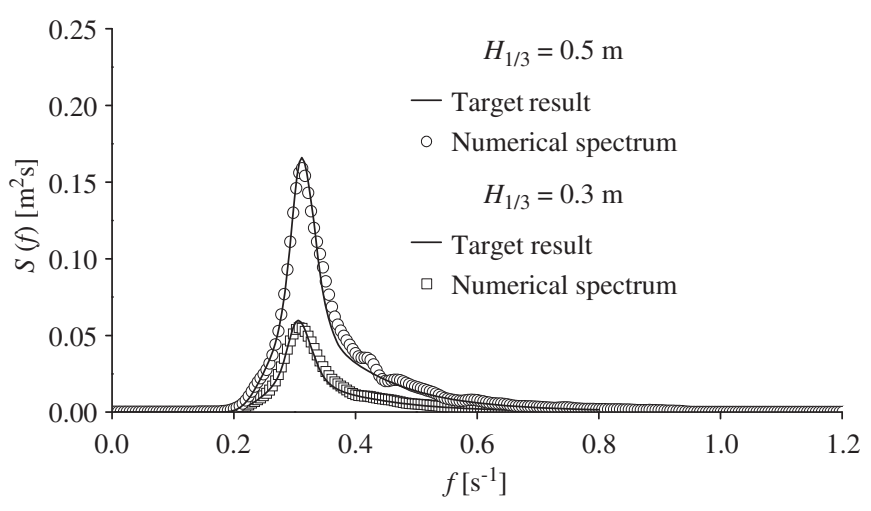

Fig. 4. Comparison of wave spectra.

$H_{1 / 3}=0.3 \mathrm{~m}$, comparison of wave spectrum at $x=4.0 \mathrm{~L}$ with the target spectrum is presented in Fig. 4. It can be seen that the incident wave spectra of two cases are consistent with the target spectra. The results indicate that the present numerical wave tank can be used to generate continuous irregular waves for a long time.

\subsubsection{Wave propagation over a submerged structure}

To verity the free surface profile during wave-structure interaction, the propagation of waves over structure in a laboratory flume (Luth et al., 1994) is considered. Fig. 5 shows the geometry of submerged structure experiment. The experiment is referred by many other researchers (Lin and Li, 2002; Janssen et al., 2006, etc.). Incident waves have period $2.86 \mathrm{~s}$ and amplitude $0.02 \mathrm{~m}$. The time step is $0.01 \mathrm{~s}$ and the computation time is carried out up to $55 \mathrm{~s}$. Fig. 6 illustrates a comparison of computed and measured free surface elevation time series at $x=20.04 \mathrm{~m}, 24.04,28.04 \mathrm{~m}$, $30.44 \mathrm{~m}, 33.64 \mathrm{~m}$ and $41.04 \mathrm{~m}$. The comparison shows a good agreement of the surface elevations, which indicates that the present model can be able to simulate the free surface correctly during wave-structure interaction.

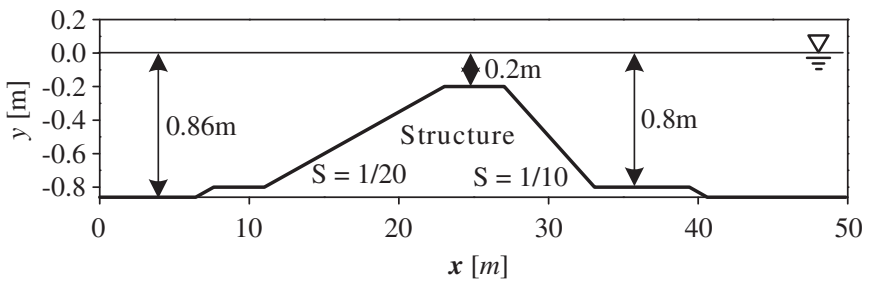

Fig. 5. Sketch of submerged structure experiment of Luth et al. (1994).

\subsubsection{Wave impact simulation}

The wave height is chosen as $0.07 \mathrm{~m}$ and $\xi$, where $\xi=\left(\omega^{2} / g\right)(B / 2)$, is chosen as $0.2,0.5,0.75,1.0,1.5$ and 1.75 . Water depth is equaled to the wave length and $h=0.25 \mathrm{~m}$, $B=0.5 \mathrm{~m}$. The results of impact simulation are compared with the experimental results of Nojiri and Murayama (1975), analytical solution of Maruo (1960), BEM results of Koo and Kim (2007a) and numerical results of $\mathrm{Li}$ and $\mathrm{Lin}$ (2010) with $k$ $\varepsilon$ model.

Figs. 7-9 show the comparison of the force components and moment, where Fx and Fy are the force component in the $x$ and $y$ direction and $M$ is the moment about the $z$ axis. $F x, F y$ and $M$ are normalized by $\rho g L h(H / 2), \rho g L B(H / 2)$ and $\rho g L h B(H / 2)$, respectively. The first-harmonic force components and rotational moment are calculated using Fourier analysis. The calculated results are shown to be in good agreement with experimental and analytical data except the region of $\xi>1.5$. Koo and Kim (2007a) considered the floating bodies based on potential theory and boundary element method (BEM). The comparison between the BEM results and present results shows that the present results based on turbulent theory are more close to experimental data than Koo and Kim's, which indicates that the turbulence of fluid has an important influence on the wave-body interaction.

The present results based on RNG model are also compared with that based on standard $k-\varepsilon$ model. It can be seen that the prediction of forces for the RNG model are slightly closer to the experimental results than the standard $k-\varepsilon$ model. The RNG approach results in a modified form of the epsilon equation, which attempts to account for the different scales of fluid motion. The comparison shows that the RNG model produces better results than the standard $k-\varepsilon$ model. Fig. 10 shows the comparison of total forces in $x$ direction for $k-\varepsilon$ model and RNG $k-\varepsilon$ model. The normalized horizontal total force calculated by RNG $k-\varepsilon$ model is slightly less than that by $k-\varepsilon$ model.

\subsection{Effect of water depth on the wave impacts}

After the validation of the numerical model, the wave forces and the rotational moments are investigated for a fixed floating structure under different water depths. Table 2 indicates the input property of water depth. The value of dimensionless $d / L$ ranges from 0.18 to 0.43 , which belongs to the finite water depth. The wave period $T$ and wave height $H$ are $3.0 \mathrm{~s}$ and $0.3 \mathrm{~m}$, respectively.

Fig. 11 shows the average and maximum normalized forces and moment on the floating structure, which obtained using the absolute values of calculated results. For regular waves, the average and maximum normalized forces and moment decrease with the increase of the dimensionless water depth. For irregular waves, the maximum forces and moment induce by irregular waves reach the values of maximum peak at $d / L=0.36$, while the average values change slightly. The maximum forces and moment induced by irregular waves are $75 \%$ greater than those induced by regular waves, while the average values are $26 \%$ lower than that induced by regular waves. 
a

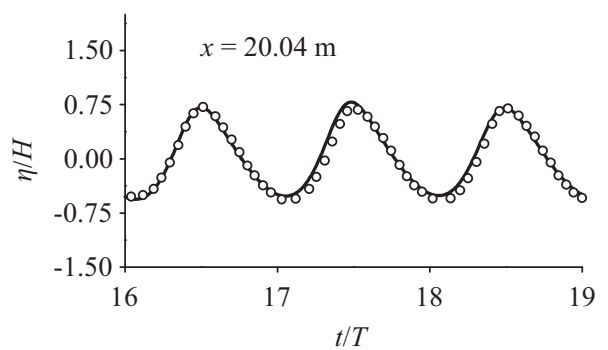

C

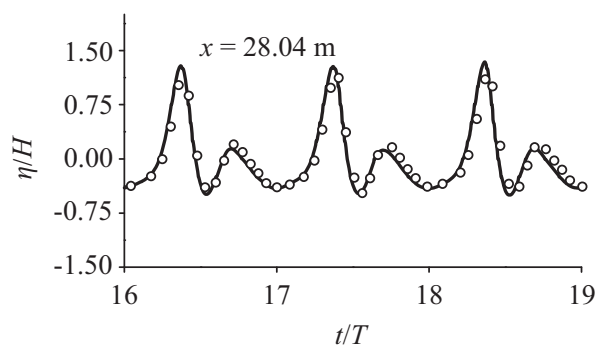

e

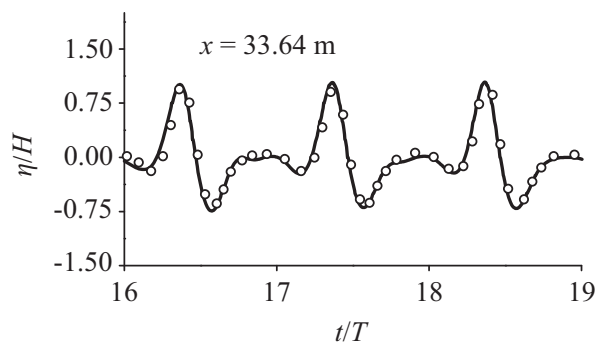

b

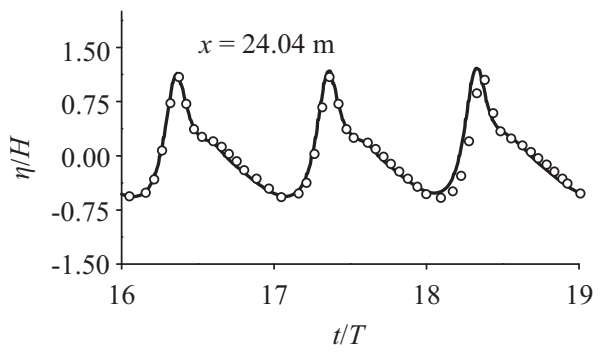

d

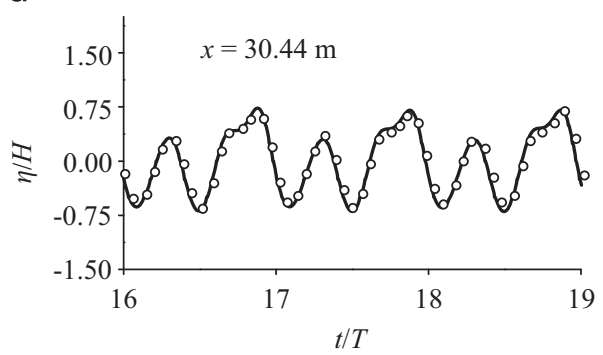

$f$

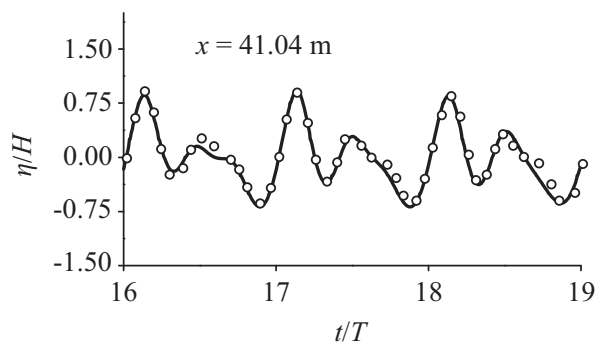

Fig. 6. Comparison of computed and measured free surface elevations over a submerged structure. Numerical solution (solid line), experimental data (circles).

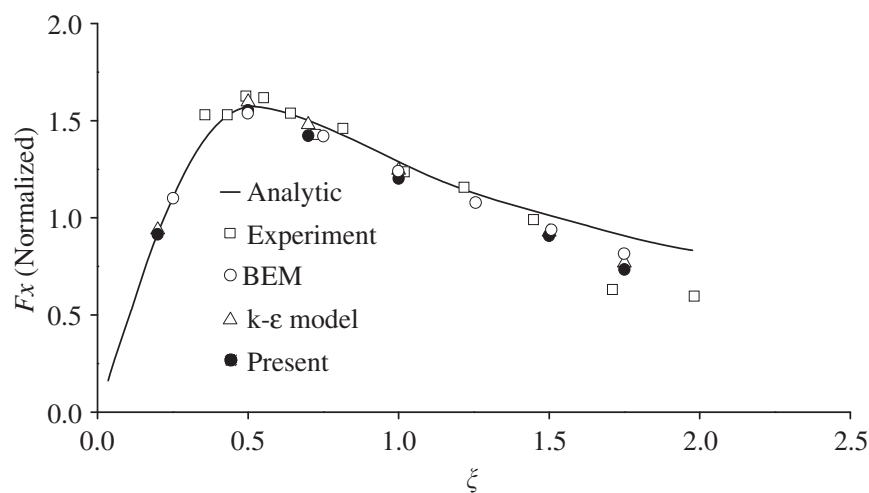

Fig. 7. Comparison of first-harmonic normalized horizontal force.

\subsection{Effect of incident wave height on the wave impacts}

For the purpose of investigating the effect of incident wave height on wave loadings, there are totally ten numerical cases are simulated for regular and irregular waves. The water depth $d$ and period $T$ are $5.0 \mathrm{~m}$ and $3.0 \mathrm{~s}$, respectively. Table 3 indicates the input property of wave height.

The simulations are performed for 200T. Partial records of $H /$ $d=0.06$ for regular and irregular waves are shown in Fig. 12. The normalized forces and moment induced by regular wave have the same frequency as the incident wave. The normalized $F y$ is less than $F x$, while it significantly larger than zero. As for the vertical

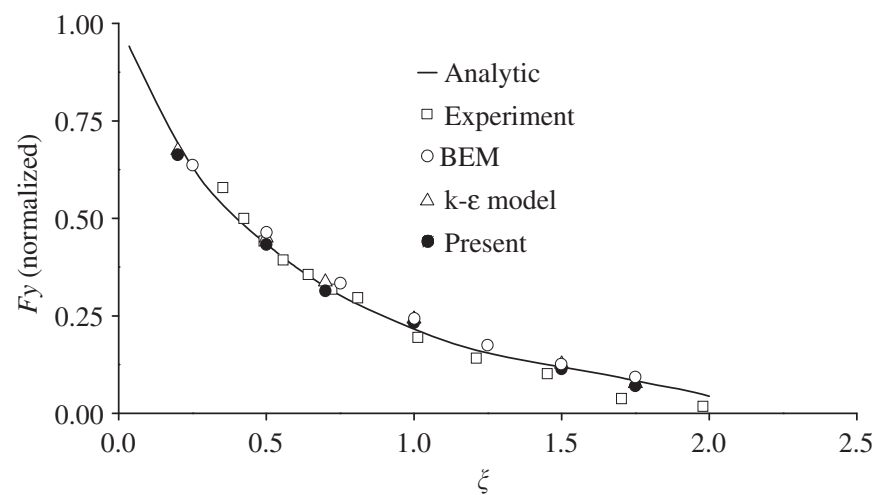

Fig. 8. Comparison of first-harmonic normalized vertical force.

structure reposed on seabed, the flow pattern is essentially symmetric about the structure in the $y$ direction and the force in this direction is close to zero ( $\mathrm{Li}$ and Lin, 2001). From Fig. 12, it can also be seen that the largest forces and moment are all induced by irregular wave.

Fig. 13 shows the average and maximum normalized forces and moment on the surface-piercing structure. The average forces and moment for regular and irregular waves and the maximum values induced by regular waves increase with the increase of the incident wave height linearly. The maximum forces and moment induced by irregular waves increase rapidly with $H / d$ and can be $100 \%$ greater than those induced by regular waves. Furthermore, 


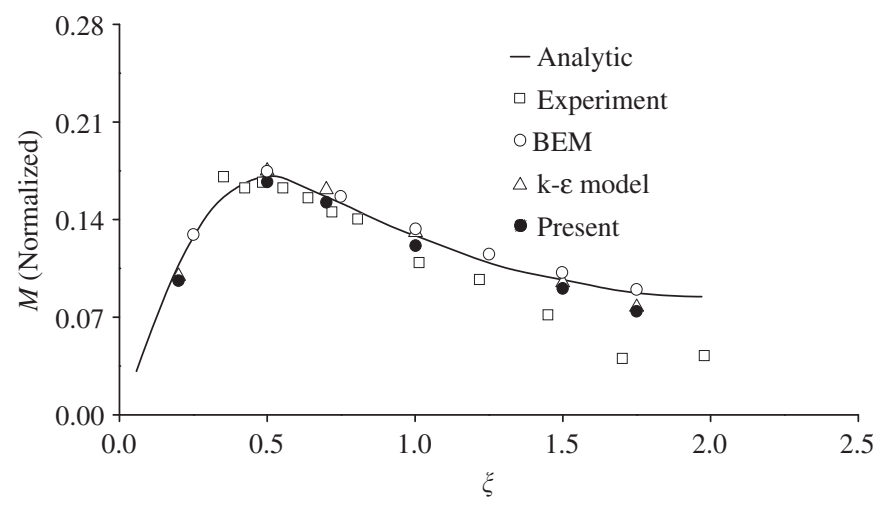

Fig. 9. Comparison of first-harmonic normalized moment.

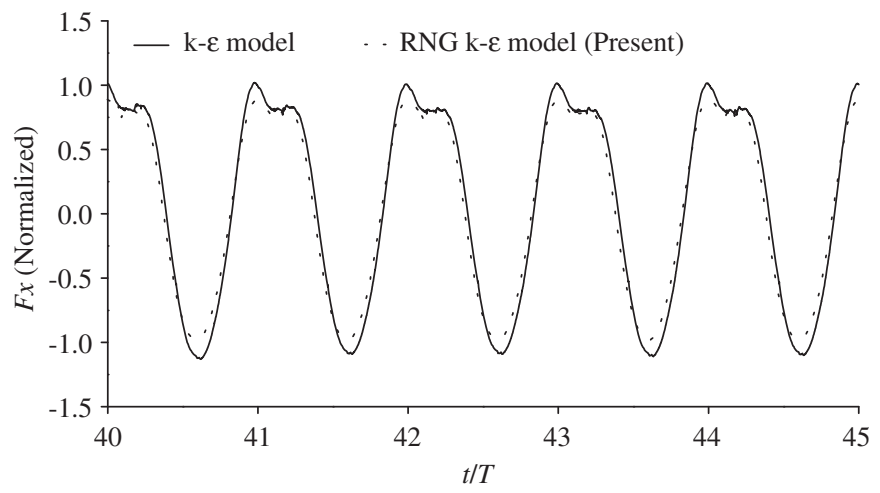

Fig. 10. Comparison of calculated total forces in the $x$ direction.

Table 2

Water depth inputs.

\begin{tabular}{llllll}
\hline Case & 1 & 2 & 3 & 4 & 5 \\
\hline$d[\mathrm{~m}]$ & 2.0 & 3.0 & 4.0 & 5.0 & 6.0 \\
$d / L$ & 0.18 & 0.24 & 0.3 & 0.36 & 0.43 \\
\hline
\end{tabular}

the average forces and moment induced by irregular waves are less than those induced by regular waves and the difference between them increases with $H / d$.

In the spectral analysis, the force and moment spectra are obtained by FFT. The amplitude spectra of the normalized forces in the $x$ and $y$ directions for irregular waves are shown in Fig. 14 . It can be seen that the spectrum of normalized $F x$ for $H / d=0.08$ has multiple peaks. The first peak frequency of the spectrum is the same as that of $H / d=0.04$. Ren and Wang (2003) found that the impact pressure spectrum had multiple peaks and the first peak frequency of the pressure spectrum was equaled to that of the incident wave spectrum.

Fig. 15 shows the comparison of amplitude spectra for normalized $F x$ between regular and irregular waves at $H / d=0.06$. The shape of the spectrum for irregular wave is basically similar to that for regular wave, while the value of first peak is less than that induced by regular wave. In addition, the energy for irregular wave is more concentrated and the spectrum of normalized $F x$ for regular wave of $H / d=0.06$ also has multiple peaks. The figure indicates that the regular wave has more energy than irregular wave under the same inputs.

\subsection{Effect of incident wave period on the wave impacts}

In this section, effect of incident wave period on the wave impacts is illustrated. The water height $H$ and water depth $d$ are
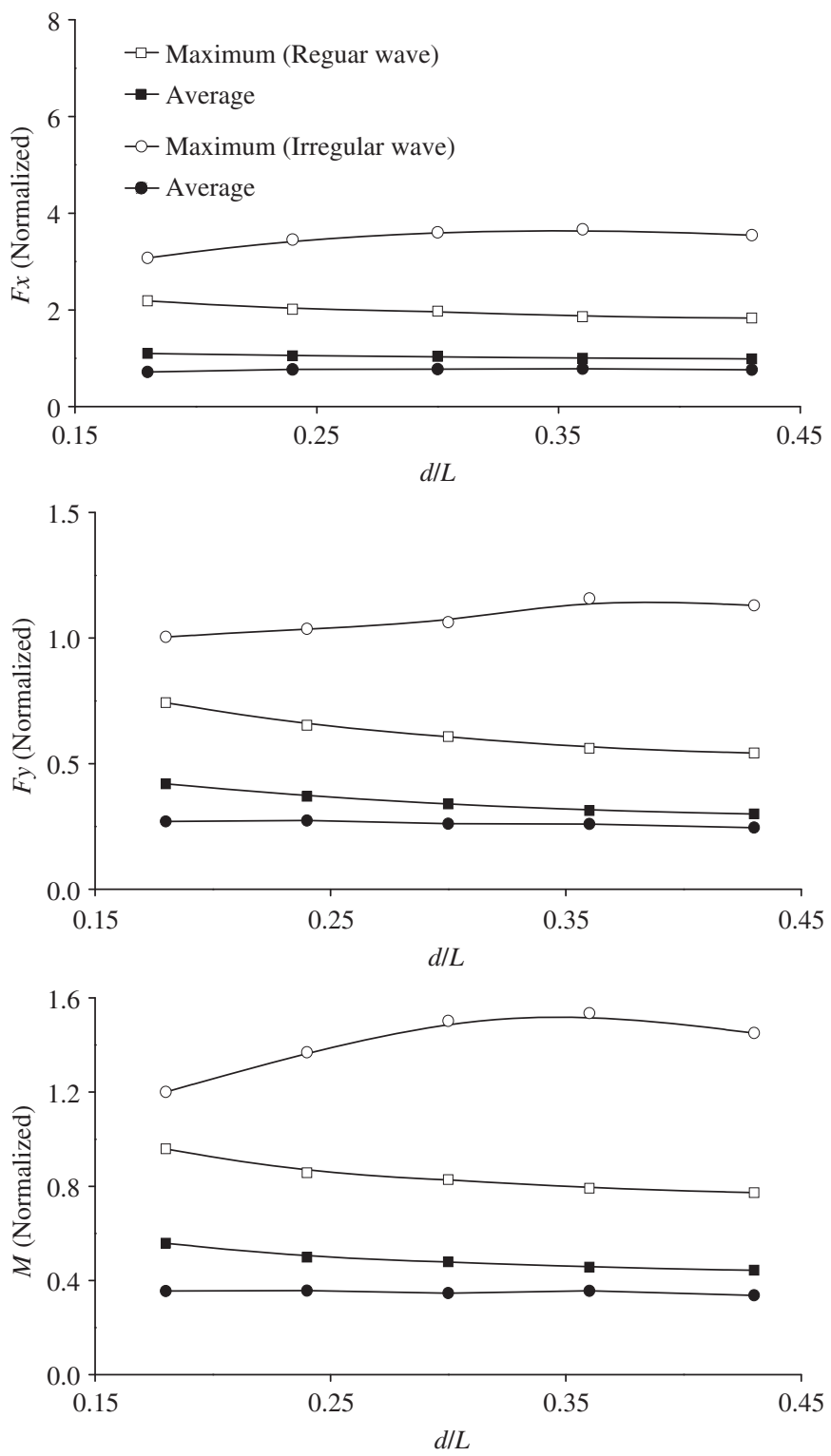

Fig. 11. Average and maximum normalized horizontal force (top), vertical force (middle) and moment (bottom) on the structure.

Table 3

Incident wave height inputs.

\begin{tabular}{llllll}
\hline Case & 1 & 2 & 3 & 4 & 5 \\
\hline$H[\mathrm{~m}]$ & 0.1 & 0.2 & 0.3 & 0.4 & 0.5 \\
$H / d$ & 0.02 & 0.04 & 0.06 & 0.08 & 0.10 \\
\hline
\end{tabular}

$0.3 \mathrm{~m}$ and $5.0 \mathrm{~m}$, respectively. Table 4 indicates the input property of wave period and the value of dimensionless period ranges from 2.8 to 5.6 .

Fig. 16 shows the average and maximum normalized forces and moment versus relative wave period. For irregular waves, the maximum normalized $F x$ reaches the value of maximum peak at $T \sqrt{g / d}=4.9$ and the magnitude gradually increases up to $150 \%$ of the value at $T \sqrt{\mathrm{g} / \mathrm{d}}=2.8$. The normalized $F x$ for regular waves and the average values for irregular waves reach the maximum peak values at about $T \sqrt{g / d}=3.5$. And then, all of them decrease slightly with the increase of dimensionless period. 

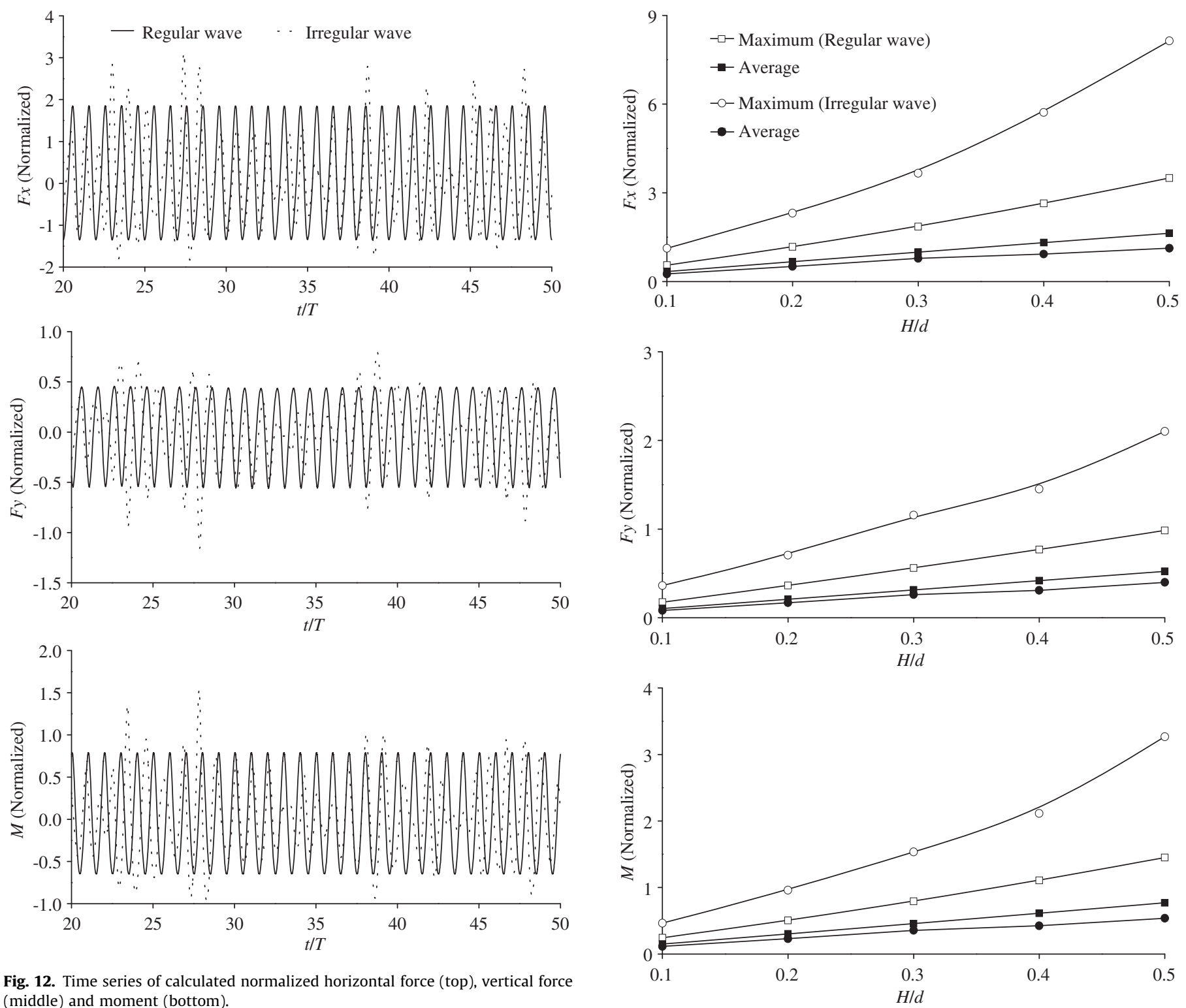

Fig. 12. Time series of calculated normalized horizontal force (top), vertical force (middle) and moment (bottom).

As for the normalized Fy, the average and maximum normalized forces for regular waves increase linearly with $T \sqrt{g / d}$, while the forces induced irregular waves increase nonlinearly. The average and maximum normalized moments increase with the increase of $T \sqrt{\mathrm{g} / \mathrm{d}}$ from 2.8 to 4.9 . The maximum moments decrease and the average values nearly keep unchanged when $T \sqrt{g / d}>4.9$.

In addition, the maximum forces and moment induced by irregular waves are much greater than that induced by regular waves and the average forces and moment induced by irregular waves are less than those induced by regular waves. For example, in case of $T \sqrt{\mathrm{g} / \mathrm{d}}=4.9$, the maximum normalized $F x$ for irregular wave is $120 \%$ greater than that for regular wave, while the average value is $21 \%$ lower than that induced by regular wave.

The amplitude spectra of the normalized $F x$ and $F y$ are shown in Fig. 17. It can be seen that the peak value of spectrum for normalized $F x$ at $T \sqrt{g / d}=4.9$ is nearly the same as that at $T \sqrt{g / d}=3.5$, while the peak of $T \sqrt{g / d}=4.9$ for normalized $F y$ is $166 \%$ greater than that of $T \sqrt{g / d}=3.5$. The wave period has a more important influence on spectrum peak of the normalized $F y$ than that of normalized $F x$.

Fig. 13. Average and maximum normalized horizontal force (top), vertical force (middle) and moment (bottom) on the structure.

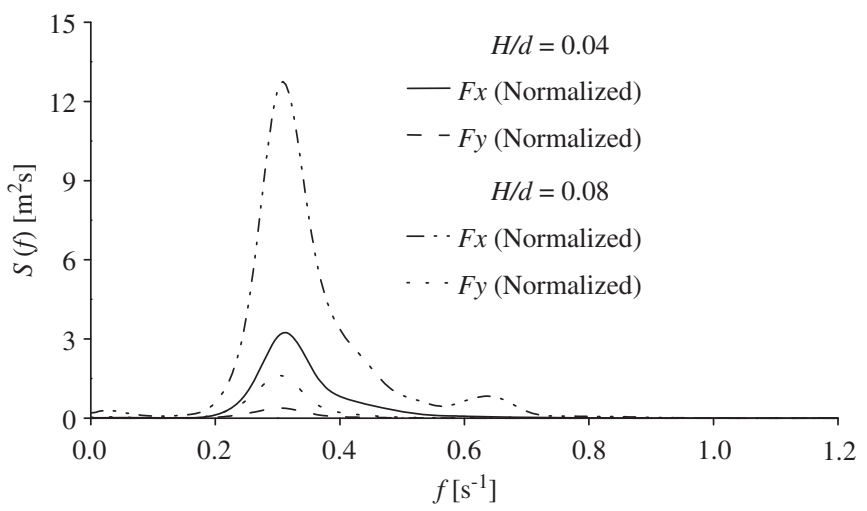

Fig. 14. Amplitude spectra of normalized forces for irregular wave. 


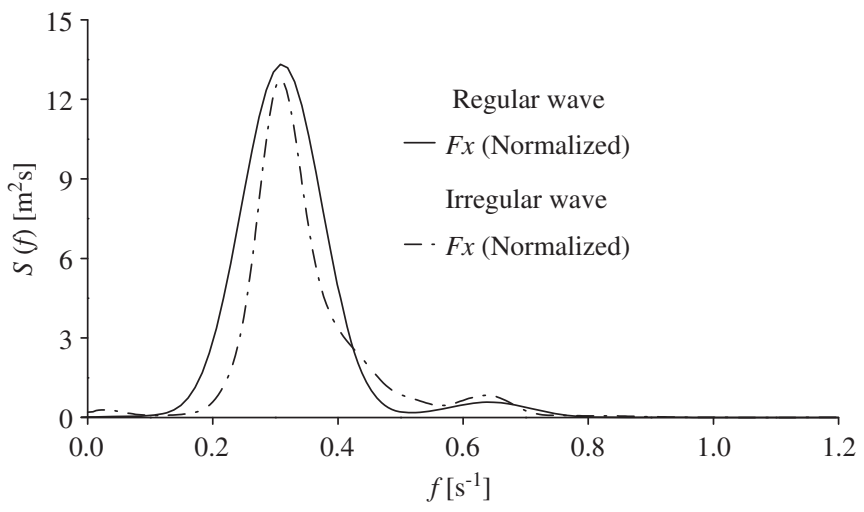

Fig. 15. Comparison of amplitude spectra between regular and irregular waves $(H / d=0.06)$

Table 4

Incident wave period inputs.

\begin{tabular}{llllll}
\hline Case & 1 & 2 & 3 & 4 & 5 \\
\hline$T[\mathrm{~s}]$ & 2.0 & 2.5 & 3.0 & 3.5 & 4.0 \\
$T \sqrt{\mathrm{g} / d}$ & 2.8 & 3.5 & 4.2 & 4.9 & 5.6 \\
\hline
\end{tabular}

Fig. 18 shows the comparison of amplitude spectra for normalized $F x$ between regular and irregular waves at $T \sqrt{g / d}=4.2$. The value of main peak for irregular wave is less than that for regular wave and the energy for irregular wave is also more concentrated.

\section{Conclusion}

In present paper, wave-body interactions for a floating structure under regular and irregular waves are simulated in a 2-D fully nonlinear numerical wave tank.

The tank model is based on RANS equations and RNG $k-\varepsilon$ model. The equations are discretized based on the FVM. The PISO scheme is employed to treat the pressure-velocity coupling and a CICSAM is used to capture the fluid interfaces on mashes of arbitrary topology. A new two-fluid code, called waveFoam, has been written within the framework of OpenFOAM. The inflow boundary and two damping zones are added into the code and the interface capturing scheme in OpenFOAM for two-phase flow is improved. The simulated results for regular wave, irregular wave and wave impacts are compared with the theoretical/experimental data. The numerical results are shown to be in good agreement with analytical/experimental solutions. Then, the mean and maximum wave impacts at different wave heights and periods are calculated and the force spectrum is obtained by FFT.

The average and maximum normalized forces and moment induced by regular waves decrease with the increase of the dimensionless water depth. For irregular waves, the maximum forces and moment induce by irregular waves reach the values of maximum peak at $d / L=0.36$, while the average values change slightly. The maximum forces and moment induced by irregular waves are much greater than those induced by regular waves, while the average values are lower than that induced by regular waves.

The average forces and moment for regular and irregular waves and the maximum values induced by regular waves increase with the increase of the incident wave height linearly. The maximum forces and moment induced by irregular waves increase rapidly with $H / d$ and the average values are less than
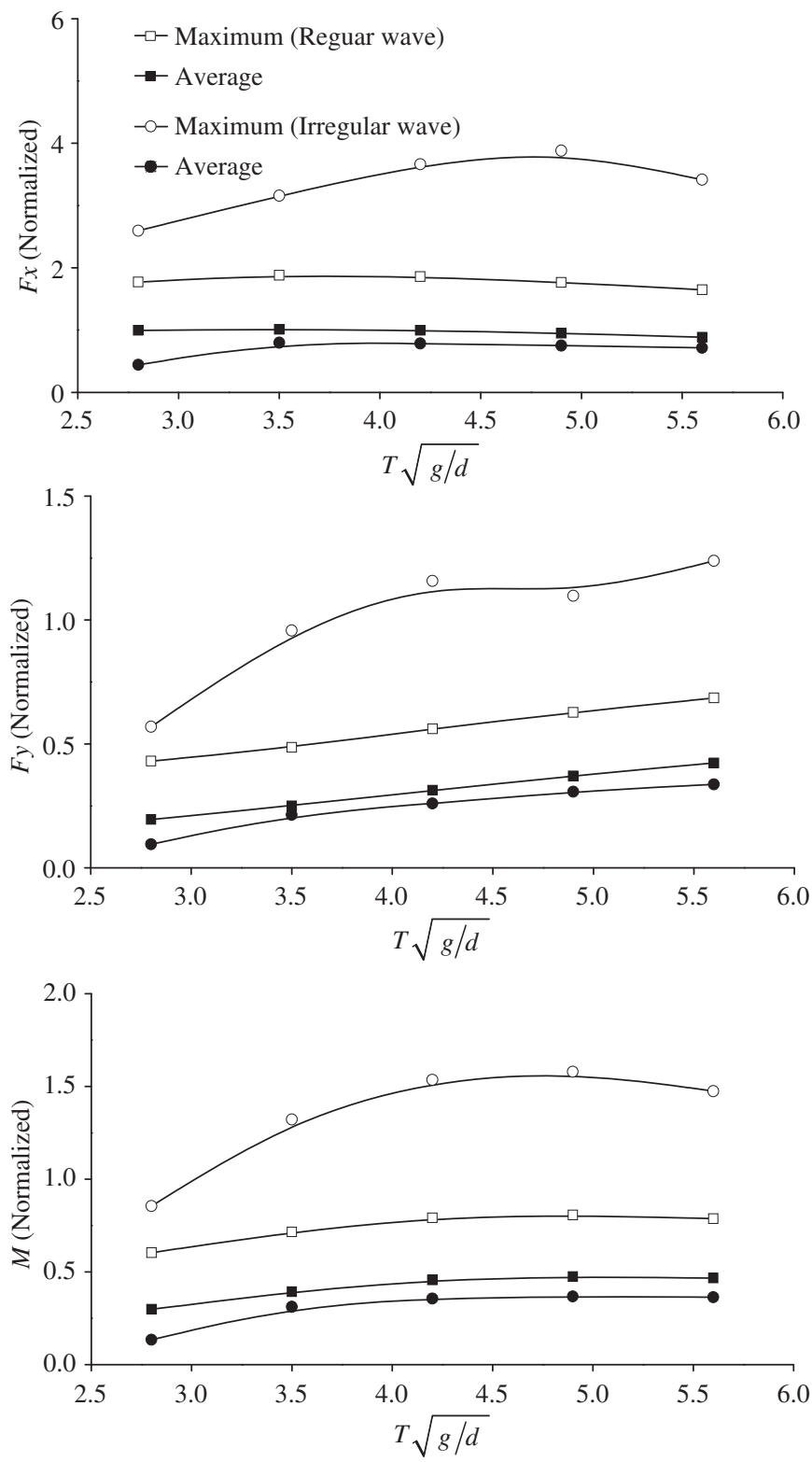

Fig. 16. Average and maximum normalized horizontal force (top), vertical force (middle) and moment (bottom).

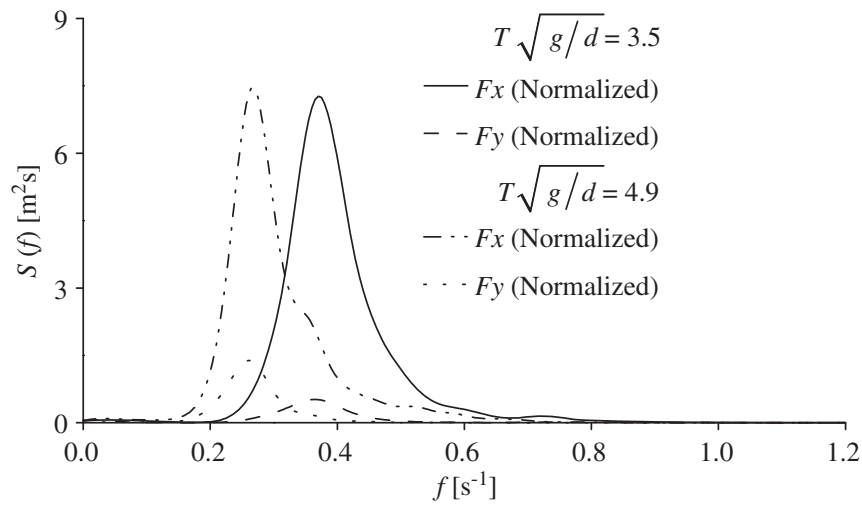

Fig. 17. Amplitude spectra of normalized forces for irregular wave. 


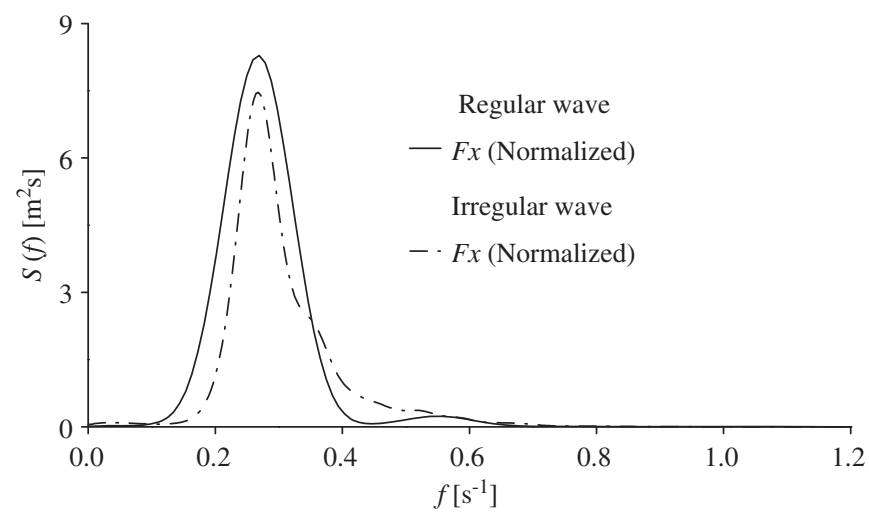

Fig. 18. Comparison of amplitude spectra between regular and irregular waves.

those induced by regular waves. The spectrum of normalized $F x$ has multiple peaks in some cases and the first peak frequency of the force spectrum is equaled to that of the incident wave spectrum. Furthermore, the value of first peak for irregular waves is less than that for regular wave under the same inputs.

As for the effect of incident wave period on the wave impacts, the maximum normalized $F x$ for irregular waves reaches the value of maximum peak at $T \sqrt{g / d}=4.9$ and the average and maximum normalized $F x$ for regular waves reach the maximum peak values at about $T \sqrt{g / d}=3.5$. The average and maximum normalized Fy for regular waves increase linearly with $T \sqrt{g / d}$, while the forces for irregular waves increase nonlinearly. The average and maximum normalized moments increase with the increase of $T \sqrt{\mathrm{g} / \mathrm{d}}$ from 2.8 to 4.9 . The maximum moments decrease and the average values nearly keep unchanged when $T \sqrt{g / d}>4.9$. In the spectral analysis, it is found that the wave period has a more important influence on spectrum peak of the normalized $F y$ than that of normalized $F x$.

\section{Acknowledgments}

The research work is supported by the National Natural Science Foundation of China (Grant no. 40776057) and the Knowledge Innovation Program of Chinese Academy of Sciences (Grant nos. KJCX2-YW-L07, KZCX2-YW-212-2).

\section{References}

Boo, S.Y., 2002. Linear and nonlinear irregular waves and forces in a numerical wave tank. Ocean Eng. 29, 475-493.
Clement, A.H., Mas, S., 1995. Hydrodynamic forces induced by solitary wave on a submerged circular cylinder. In: Proceedings of the Fifth International Offshore and Polar Engineering Conference, ISOPE, The Hague 3, pp. 339-347.

Fang, M.C., Chen, G.R., 2001. The relative motion and wave elevation between two floating structures in waves. In: Proceedings of the Eleventh International Offshore and Polar Engineering Conference, ISOPE, Stavanger 3, pp. 361-368.

Goda, Y., 1999. A comparative review on the functional forms of directional wave spectrum. Coastal Eng. J. 41 (1), 1-20.

Hanssen, A.G., Torum, A., 1999. Breaking wave forces on tripod concrete structure on shoal. J. Water Way Port Coast Ocean Eng. 125 (6), 304-310.

Janssen, T.T., Herbers, T.H.C., Battjes, J.A., 2006. Generalized evolution equations for nonlinear surface gravity waves over two-dimensional topography. J. Fluid Mech. 552, 393-418.

Koo, W.C., Kim, M.H., Tavassoli, A., 2004. Fully nonlinear wave-body interactions with fully submerged dual cylinders. Int. J. Offshore Polar Eng. 14 (3), 210-217.

Koo, W.C., Kim, M.H., 2007a. Fully nonlinear wave-body interactions with surfacepiercing bodies. Ocean Eng. 34, 1000-1012.

Koo, W.C., Kim, M.H., 2007b. Current effects on nonlinear wave-body interactions by a $2 \mathrm{D}$ fully nonlinear numerical wave tank. J. Water Way Port Coast Ocean Eng. 133 (2), 136-146.

Li, B., 2008. A 3-D model based on Navier-Stokes equations for regular and irregular water wave propagation. Ocean Eng. 35, 1842-1853.

Li, C.W., Lin, P.Z., 2001. A numerical study of three-dimensional wave interaction with a square cylinder. Ocean Eng. 28, 1545-1555.

Li, L., Lin, Z.W., You, Y.X., Mao, G.P., 2007. The numerical wave flume of the viscous fluid based on the momentum source method. J. Hydrodyn. Ser. A 22 (1), 76-82.

Li, Y., Lin, M., 2010. Wave-body interactions for a surface-piercing body in water of finite depth. J. Hydrodyn. Ser. B 22 (6), 745-752.

Lin, P., Li, C.W., 2002. A $\alpha$-coordinate three-dimensional numerical model for surface wave propagation. Int. J. Numer. Methods Fluids 38 (11), 1045-1068.

Luth, H.R., Klopman, G., Kitou, N., 1994. Project 13G: Kinematics of Waves Breaking Partially on an Offshore Bar: LDV Measurements for Waves with and without a Net Onshore Current. Delft Hydraulics Report H1573. Delft Hydraulics, The Netherlands.

Maruo, H., 1960. On the increase of the resistance of a ship in rough seas. J. Zosen Kiokai, 108.

Nojiri, N., Murayama, K., 1975. A study on the drift force on two dimensional floating body in regular waves. Trans. West-Jpn. Soc. Nav. Archit. 51, 131-152.

Pradip, D.R., Sukamal, G., 2006. Wave force on vertically submerged circular thin plate in shallow water. Ocean Eng. 33, 1935-1953.

Ren, B., Wang, Y.X., 2003. Experimental study of irregular wave impact on structures in the splash zone. Ocean Eng. 30, 2363-2377.

Scanlon, T.J., Roohi, E., White, C., Darbandi, M., Reese, J.M., 2010. An open source, parallel DSMC code for rarefied gas flows in arbitrary geometries. Comput. Fluids 39, 2078-2089.

Tanizawa, K., Minami, M., 1998. On the accuracy of NWT for radiation and diffraction problem. In: Proceedings of the Abstact for the Sixth Symposium on Nonlinear and Free-surface Flow.

Troch P., Rouck J., 1998. Development of two-dimensional numerical wave flume for wave interaction with rubble mound breakwaters. In: Proceedings of Twenty-sixth Conference on Coastal Engineering, pp. 1638-1649.

Ubbink, O., Issa, R.I., 1999. A method for capturing sharp fluid interfaces on arbitrary meshes. J. Comput. Phys. 153 (1), 26-50.

Vengatesan, V., Varyani, K.S., Barltrop, N.D.P., 2006. Wave force coefficients for horizontally submerged rectangular cylinders. Ocean Eng. 33, 1669-1704.

Wang, C.Z., Wu, G.X., 2010. Interactions between fully nonlinear water waves and cylinder arrays in a wave tank. Ocean Eng. 37, 400-417.

Zhang, X.T., Khoo, B.C., Lou, J., 2006. Wave propagation in a fully nonlinear numerical wave tank: a desingularized method. Ocean Eng. 33, 2310-2331. 